J. Nonlinear Var. Anal. 2 (2018), No. 2, pp. 155-164

Available online at http://jnva.biemdas.com

https://doi.org/10.23952/jnva.2.2018.2.04

\title{
STURM THEOREMS AND DISTANCE BETWEEN ADJACENT ZEROS FOR SECOND ORDER INTEGRO-DIFFERENTIAL EQUATIONS
}

\author{
ALEXANDER DOMOSHNITSKY
}

Department of Mathematics, Ariel University, Ariel 40700, Israel

\begin{abstract}
Between two adjacent zeros of any nontrivial solution of the second order ordinary differential equation $x^{\prime \prime}(t)+$ $a(t) x^{\prime}(t)+b(t) x(t)=0$ there is one and only one zero of every nonproportional solution. This principle of zeros' distribution is known as the Sturm separation theorem which is a basis of many classical results on oscillation and asymptotic properties and on boundary value problems for ordinary differential equations. For delay and integro-differential equations this principle of zeros' distribution is not true. In this paper, the assertion on validity of the Sturm separation theorem are proposed. Distance between two zeros of nontrivial solutions to integro-differential equations is estimated.
\end{abstract}

Keywords. Boundary value problem; Integro-differential equation; Sturm separation theorem; Distance between zeros

2010 Mathematics Subject Classification. 34K20.

\section{INTRODUCTION}

Oscillation and asymptotic properties of the delay equation

$$
\begin{gathered}
x^{\prime \prime}(t)+q(t) x^{\prime}(t-\theta(t))+p(t) x(t-\tau(t))=0, \quad t \in[0,+\infty), \\
x(\xi)=\varphi(\xi), x^{\prime}(\xi)=\varphi^{\prime}(\xi) \text { for } \xi \leq 0,
\end{gathered}
$$

were considered in the classical monographs by Erbe, Kong and Zhang [11], Gyori and Ladas [12], Ladde, Lakshmikantham and Zhang [20], Myshkis [21], Norkin [22]. It should be stressed that unlike ordinary differential equations, the delay equation (1.1) can have both oscillating and nonoscillating solutions. For example, the equation

$$
x^{\prime \prime}(t)-2 x(t-\tau(t))=0, \quad t \in[0,+\infty),
$$

with

$$
\tau(t)=\left\{\begin{array}{lc}
t, & t \in[0,4), \\
t-2, & t \in(4,8),
\end{array} \quad \tau(t+8)=\tau(t),\right.
$$

possesses oscillating periodic solution

$$
x(t)=\left\{\begin{array}{cl}
(t-1)(t-3), & t \in[0,4], \\
-(t-4)^{2}+4(t-4)+3, & t \in(4,8),
\end{array} \quad x(t+8)=x(t),\right.
$$

and a group of nonoscillation solutions $y(t)$ which satisfy the conditions $y(0)=\alpha \geq 0, \quad y^{\prime}(0)=\beta>0$. It is clear that $y^{\prime \prime}(t) \geq 0, y^{\prime}(t)>0$ for $t \in[0,+\infty)$ that implies $y(t)>0$ for $t \in[0,+\infty)$, and $y(t) \rightarrow+\infty$.

E-mail address: adom@ariel.ac.il.

Received February 28, 2018; Accepted April 20, 2018.

(C)2018 Journal of Nonlinear and Variational Analysis 
Sufficient conditions of oscillation of all solutions were proposed in [15] (see also the bibliography therein and the paper [16]). The distance between zeros of solutions to second order equation (1.1) has been studied since the classical book by Myshkis [21]. Various estimates of the distance between two adjacent zeros of nontrivial solutions to delay equation (1.1) were obtained in $[2,4,7,9,10,14,19,21$, 24]. Applications of distances between zeros to the study of the size of zones of positivity of solutions of partial delay differential equations were proposed in [8]. For ordinary homogeneous differential equation $x^{\prime \prime}(t)+a(t) x^{\prime}(t)+b(t) x(t)=0$, there is the following principle in distribution of zeros, called the Sturm separation theorem: between two adjacent zeros of each nontrivial solution there is one and only one zero of every linearly independent solution. The Sturm separation theorem follows from non-vanishing Wronskian

$$
W(t)=\left|\begin{array}{ll}
x_{1}(t) & x_{2}(t) \\
x_{1}^{\prime}(t) & x_{2}^{\prime}(t)
\end{array}\right|
$$

of a fundamental system $\left\{x_{1}(t), x_{2}(t)\right\}$ of this ordinary differential equation. Indeed, let us suppose the existence of two zeros $t_{1}$ and $t_{2}$ of nontrivial solution $x_{2}$ between adjacent zeros of $x_{1}$. Consider the following function $y(t)=\frac{x_{2}(t)}{x_{1}(t)}$. From the form of $y(t)$, it follows that $y\left(t_{1}\right)=y\left(t_{2}\right)=0$, but this contradicts to the fact that the derivative $y^{\prime}(t)=\frac{W(t)}{\left[x_{1}(t)\right]^{2}}$ preserves its sign for $t \in\left[t_{1}, t_{2}\right]$.

What are homogeneous equation, fundamental systems and the Wronskian in the case of equation (1.1)? If $\varphi$ in (1.2) is a fixed twice differentiable function, only the Cauchy problem can be considered and a notion of the fundamental system does not appear. If equation (1.1), (1.2) is studied for all possible twice differentiable functions $\varphi$, the space of its solutions is infinity-dimensional. Consider, for example, the equation

$$
x^{\prime \prime}(t)+x(t-\pi)=0, \quad t \in[0,+\infty) .
$$

The function $x=\sin n t$ satisfies (1.3) for the initial function

$$
x(\xi)=n^{2} \sin (n \xi) \text { for } \xi<0 .
$$

Thus there exists a solution with every number $n$ of zeros on the interval $[0, \pi]$. Examples of this sort demonstrate that there are no nonoscillation intervals (i.e. intervals, where solution of equation (1.3) has at most one zero) and the Sturm separation theorem is not true for such defined homogeneous delay differential equations. As a result, in the classical books on oscillation of delay equations [11, 12, 20, 21] noted above, nonoscillation for delay differential equations meant only the existence of eventually positive solutions on the semiaxis $t \in(0, \infty)$. The absence of the concept of a finite nonoscillation interval does not allow to use the well developed (in the frame of the theory of ordinary differential equations) nonoscillation technique to delay differential equation (1.1).

Azbelev in his paper [2] avoided a historical fully formed tradition to consider a solution of delay equation (1.1) as a continuously prolonged initial function $\varphi(t)$ and defined a homogeneous delay equation as equation (1.1) with the zero initial functions

$$
x(\xi)=0, x^{\prime}(\xi)=0 \text { for } \xi<0 .
$$

The space of its solutions becomes two-dimensional, the fundamental systems $\left\{x_{1}(t), x_{2}(t)\right\}$ and the Wronskian $W(t)$ can be considered and methods of the classical theory of linear ordinary differential equations based on the analysis of the fundamental systems work also for delay differential equation $(1.1)$. 
Generally speaking, the Sturm separation theorem is not valid for delay differential equations even in the case of zero initial function defined by (1.5). The first results on nonvanishing Wronskian on the semiaxis $[0,+\infty)$ and consequently on validity of the Sturm separation theorem for the delay differential equation

$$
\begin{gathered}
x^{\prime \prime}(t)+\sum_{i=1}^{m} p_{i}(t) x\left(h_{i}(t)\right)=0, p_{i}(t) \geq 0, t \in[0, \infty), \\
x(\xi)=0 \text { for } \xi<0
\end{gathered}
$$

were obtained in [2] in the form of conditions on a corresponding smallness of the delays. Denote $h(t)=\min _{1 \leq i \leq m} h_{i}(t)$.

Theorem 1.1. [2]. If $(t-h(t)) \int_{h(t)}^{t} \sum_{i=1}^{m} p_{i}(s) d s \leq 4$ or $(t-h(t))^{2} \operatorname{esssup}_{h(t) \leq s \leq t} \sum_{i=1}^{m} p_{i}(s) \leq 8$ for a.e. $t \geq 0$, then $W(t) \neq 0$ for $t \geq 0$.

These results were extent to the neutral delay differential equation

$$
x^{\prime \prime}(t)-q(t) x^{\prime \prime}(g(t))+\sum_{i=1}^{m} p_{i}(t) x\left(h_{i}(t)\right)=0, p_{i}(t), q(t) \geq 0, t \in[0, \infty),
$$

with the initial function defined by (1.7) in the paper [4]. A result of another type based on specific properties of the binomial equation

$$
\begin{gathered}
x^{\prime \prime}(t)+p(t) x(h(t))=0, p(t) \geq 0, t \in[0, \infty), \\
x(\xi)=0 \text { for } \xi<0,
\end{gathered}
$$

with nondecreasing $h(t)$ was obtained in $[18,19]$.

Theorem 1.2. If the function $h(t)$ is nondecreasing, then $W(t) \neq 0$ for $t \geq 0$.

The following question can be formulated: what are the properties of the operator $Q$ in order to preserve the distribution of zeros of solutions of the binomial equation (1.9) for the equation

$$
x^{\prime \prime}(t)+(Q x)(t)=0, t \in[0, \infty) ?
$$

In the paper [7], an answer was obtained for the operator $(Q x)(t)=\sum_{i=1}^{m} p_{i}(t) x\left(h_{i}(t)\right)$ with nondecreasing $h_{i}(t)$, small differences $\left|h_{i}(t)-h_{j}(t)\right|$ and several additional assumptions on the coefficients $p_{i}(t)$, in this paper - for the operator $(Q x)(t)=\int_{h(t)}^{\Delta(t)} K(t, s) x(s) d s$, where $K(t, s) \geq 0, h(t)$ and $\Delta(t)$ are nondecreasing functions.

Note another approach to the Sturm separation theorem in non-standard form developed in the paper [9]. In [21], the idea of semi-cycles and big semi-cycles for equation (1.1) was proposed. An interval $[v, \mu]$ is a semi-cycle if $x(v)=x(\mu)=0, x(t) \neq 0$ for $t \in(v, \mu)$. Let $\gamma(v)$ be a such number that $h_{i}(t) \geq v$ for $t \geq \gamma(v)$. If $\gamma(v)<\mu$, we call this interval big semi-cycle. If $[v, \mu]$ is a big semi-cycle of (1.1), then the interval $\left[v^{*}, \mu\right]$, where $v^{*}=\operatorname{essinf}_{t \in[v, \mu]} h(t), h(t)=\min _{1 \leq i \leq m} h_{i}(t)$ was called the extent big semicycle. In [9], the following variant of the Sturm separation theorem for delay differential equation (1.1) is proposed: if for a solution $x(t)$ the interval $\left[v^{*}, \mu\right]$ is an extent big semi-cycle, then each solution $y(t)$ has zero in $\left[v^{*}, \mu\right]$.

For ordinary differential equations, the growth of the Wronskian and unboundedness of solutions on this basis are obtained in the well known book [13]. If the period $\omega$ of the coefficient $b(t)$ such 
that $\int_{0}^{\omega} b(t) d t>0$ in the equation $x^{\prime \prime}(t)+b(t) x(t)=0$ less than the distance between adjacent zeros of solutions, then all its solutions are bounded [25]. An analysis of the properties of the Wronskian allows to construct a similar technique for delay differential equations. Results on existence of unbounded solutions based on the growth of the Wronskian $W(t)$ were obtained in the papers $[5,6,23]$ for delay differential equation (1.1). A combination of results on the growth of the Wronskian and estimates of distances between zeros of solutions to equation (1.1) leads to assertions on unboundedness of all solutions [6].

In this paper, the Sturm separation theorem and results on estimates of the distance between zeros of solutions are proposed for the integro-differential equation

$$
x^{\prime \prime}(t)+\int_{h(t)}^{\Delta(t)} K(t, s) x(s) d s=0, K(t, s)>0, t, s \in[0, \infty) .
$$

It is assumed that $h(t)$ and $\Delta(t)$ are nonnegative nondecreasing measurable functions, $h(t) \leq \Delta(t)$ for $t \in[0, \infty)$. We find conditions on the kernel $K(t, s)$ such that integro-differential equation (1.11) preserves similar oscillation properties as in the case of binomial equation (1.9). An analogue of Theorem 1.2 for equation (1.11) will be proposed. The results are based on nonvanishing Wronskian. Note that the condition $W(t) \neq 0$ for $t \in[0, \infty)$ is equivalent to the fact that the one-point boundary value problem consisting equation (1.11) with the conditions $x(\omega)=0, x^{\prime}(\omega)=0$ has only the trivial solution $x(t) \equiv 0$ for $t \in[0, \omega]$ for each $\omega \in(0, \infty)$ (see the general theory of functional differential equations [3]). This can be interpreted also in the form: nontrivial solutions of (1.11) cannot have the multiple zeros.

\section{AuXiliary LEMMAS}

Introduce the auxiliary operator $K_{v \mu}: \mathscr{C}_{[v, \mu]} \mapsto \mathscr{C}_{[v, \mu]}$ by the following equality

$$
\begin{aligned}
& \left(K_{v \mu} y\right)(t)=-\int_{v}^{\mu} G_{v \mu}(t, s) \int_{h(s)}^{\Delta(s)} K(s, \xi) y(\xi) d \xi d s, \\
& \text { where } y(\xi)=0 \text { for } \xi<v,
\end{aligned}
$$

where $y \in \mathscr{C}_{[v, \mu]}$,

$$
G_{v \mu}(t, s)= \begin{cases}-\frac{(\mu-t)(s-v)}{\mu-v}, & v \leq s \leq t \leq \mu, \\ -\frac{(t-v)(\mu-s)}{\mu-v}, & v \leq t<s \leq \mu,\end{cases}
$$

is the Green's function of boundary value problem

$$
x^{\prime \prime}(t)=f(t), t \in[v, \mu], \quad x(v)=0, \quad x(\mu)=0 .
$$

It is clear form the definition of the operator $K_{v \mu}: \mathscr{C}_{[v, \mu]} \mapsto \mathscr{C}_{[v, \mu]}$ and the condition $K(t, s)>0$ that this operator is positive. Denote $\rho\left(K_{v \mu}\right)$ its spectral radius. Let us note several known assertions about estimates of the spectral radii (see the book [17], Chapter 2, paragraph 5).

Lemma 2.1. If there exists a continuous function $v(t)$ such that $v(t)>0, v(t)>\left(K_{v \mu} v\right)(t)$ for $t \in[v, \mu]$, then $\rho\left(K_{v \mu}\right)<1$. 
Lemma 2.2. If there exists a continuous function $v(t)$ such that $v(t) \gtreqless 0, v(t) \leq\left(K_{v \mu} v\right)(t)$ for $t \in[v, \mu]$, then $\rho\left(K_{v \mu}\right) \geq 1$.

Remark 2.1. We understand $v(t) \supsetneqq 0$ as the following: $v(t) \geq 0$ for $t \in[v, \mu]$ and there exists the set $\Omega \subset[v, \mu]$ such that $v(t)>0$ for $t \in \Omega$, and the measure of such points $t$ is positive, i.e. mes $\Omega>0$.

Define the operator $K_{\alpha \beta}: \mathscr{C}_{[\alpha, \beta]} \mapsto \mathscr{C}_{[\alpha, \beta]}$ setting $\alpha$ and $\beta$ instead of $v$ and $\mu$ respectively in formulas (2.1)-(2.3). It is clear from the condition $K(t, s)>0$ that this operator is also positive. The following assertion was proven in [4].

Lemma 2.3. Assume that $v \leq \alpha \leq \beta \leq \mu$. If $\rho\left(K_{v \mu}\right)<1$, then $\rho\left(K_{\alpha \beta}\right)<1$. If $\rho\left(K_{\alpha \beta}\right) \geq 1$, then $\rho\left(K_{v \mu}\right) \geq 1$.

Lemma 2.4. If $\rho\left(K_{h(t) t}\right)<1$ for $t \geq 0$ and $\rho\left(K_{v \mu}\right) \geq 1$, then $h(t)>v$ for almost all $t>\mu$.

Proof. Let us assume in the contrary that there exists $\Omega$ such that mes $\Omega>0$ and $h(t) \leq v$ for $t \geq \mu$. Then according to Lemma 2.3, we obtain $\rho\left(K_{h(t) t}\right) \geq 1$. This contradiction proves Lemma 2.4.

\section{STURM SEPARATION THEOREM FOR INTEGRO-DIFFERENTIAL EQUATION}

Consider the equation

$$
x^{\prime \prime}(t)+\int_{h(t)}^{\Delta(t)} K(t, s) x(s) d s=0, K(t, s)>0, t, s \in[0, \infty),
$$

where $h(t)$ and $\Delta(t)$ are nonnegative nondecreasing measurable functions, $h(t) \leq \Delta(t)$ for $t \in[0, \infty)$. Concerning the kernel $K(t, s)$, the standard conditions for the action and compactness of the integral operator $(K x)(t) \equiv \int_{h(t)}^{\Delta(t)} K(t, s) x(s) d s: L_{p}[0, \omega] \rightarrow L_{p}[0, \omega]$ (see, for example, Theorem A.4, p.459 [1]) for every $\omega \in(0, \infty)$ are assumed.

Define the function

for

$$
A(t, \tau, s)=\frac{K(t+\tau, s)}{K(t, s)}
$$

$$
t \in[0, \infty), s \in[h(t), \Delta(t)], \text { and } \tau \geq 0 \text { such that } s \in[h(t+\tau), \Delta(t+\tau)] .
$$

In order to obtain oscillation properties similar to corresponding ones of binomial equation (1.9) we require that the function $A(t, \tau, \cdot)$ is nondecreasing as a function of the third variable $s$ for every possible $t$ and $\tau$ defined in (3.3).

Let us demonstrate examples of such kernels.

Example 3.1. Let us demonstrate that the function $A(t, \tau, \cdot)$ is nondecreasing for the kernel $K(t, s)=$ $\varphi(t)+\psi(s)$, where

a) $\varphi(t)$ is nondecreasing and $\psi(s)$ is nonincreasing functions,

or

b) $\varphi(t)$ is nonincreasing and $\psi(s)$ is nondecreasing functions.

Actually, let us find the derivative

$$
A_{s}^{\prime}(t, \tau, s)=\frac{\psi^{\prime}(s)[\varphi(t)-\varphi(t+\tau)]}{[\varphi(t)+\psi(s)]^{2}} \geq 0 .
$$


Example 3.2. The function $A(t, \tau, \cdot)$ is nondecreasing for the kernel $K(t, s)=\varphi_{1}(t) \psi_{1}(s)+\varphi_{2}(t)$, where a) $\varphi_{1}(t), \psi_{1}(s)$ are nondecreasing, $\varphi_{2}(t)$ is nonincreasing functions, or

b) $\varphi_{1}(t), \psi_{1}(s)$ are nonincreasing, $\varphi_{2}(t)$ is nondecreasing functions.

Let us find the derivative

$$
A_{s}^{\prime}(t, \tau, s)=\frac{\varphi_{1}(t+\tau) \varphi_{2}(t)-\varphi_{2}(t+\tau) \varphi_{1}(t)}{\left[\varphi_{1}(t) \psi_{1}(s)+\varphi_{2}(t)\right]^{2}} \psi_{1}^{\prime}(s) \geq 0 .
$$

Example 3.3. The function $A(t, \tau, \cdot)$ is nondecreasing for the kernel $K(t, s)=(t-s)^{\gamma}$, where $\gamma \geq 1$.

Theorem 3.1. Let the function $A(t, \tau, \cdot)$ be nondecreasing for $t$ and $\tau$ in the domain defined by (3.3) and the spectral radius $\rho\left(K_{h(t) \Delta(t)}\right)$ of the operator $K_{h(t) \Delta(t)}: C_{h(t) \Delta(t)} \rightarrow C_{h(t) \Delta(t)}$ be less than one for a.e. $t \in(0, \infty)$. Then

1) the Sturm separation theorem is valid for integro-differential equation (3.1);

2) if $v$ and $\mu$ are two zeros of the solution $x(t)$ of equation (3.1), then $\rho\left(K_{v \mu}\right) \geq 1$;

3) if $\rho\left(K_{v \mu}\right)<1$, then there is at most one zero of every nontrivial solution $x(t)$ on the interval $[v, \mu]$.

The following example demonstrates that the nondecreasing $A(t, \tau, \cdot)$ is essential condition in Theorem 3.1 .

Example 3.4. Consider equation (3.1), where

$$
\begin{gathered}
K(t, s)= \begin{cases}0.001, & s \in[0,1], t \in[2,503), \\
0.002, & s \in[1,2], t \in[2,503], \\
\frac{0.002}{250999}, & s \in[0,1], t \in[503, \infty), \\
\frac{0.001}{250999}, & s \in[1,2], t \in[503, \infty), \\
0, & s \notin[0,2], \\
0, & t \notin[2, \infty),\end{cases} \\
h(t) \equiv 0, \Delta(t)= \begin{cases}0, & t \in[0,2], \\
2, & t \in(2, \infty) .\end{cases}
\end{gathered}
$$

In this case, we have

$$
A(t, \tau, s)= \begin{cases}\frac{2}{250999}, & s \in[0,1], t \geq 2, \tau \geq 501 \\ \frac{1}{250999}, & s \in[1,2], t \geq 2, \tau \geq 501\end{cases}
$$

which does not satisfy the nondecreasing condition.

The function

$$
x(t)= \begin{cases}1-t, & t \in[0,2], \\ 0.001 t^{2}-1.004 t+1.004, & t \in(2,503], \\ -\frac{0.001}{250999}(251502-t)^{2}, & t \in(503, \infty),\end{cases}
$$

is a solution of (3.1) with coefficients satisfying (3.6) and (3.7) has multiple zero at the point $t=251502$, consequently $W(251502)=0$ and the Sturm separation theorem is not valid. 


\section{ProOfS}

Let us start the proof of Theorem 3.1 with the following auxiliary assertion.

Lemma 4.1. Let the function $A(t, \tau, \cdot)$ be nondecreasing for $t$ and $\tau$ in the domain defined by (3.3) and let $y(t)$ be a continuous function such that $y(t)<0$ for $t<a, y(t)>0$ for $t>a$, and there be the set $\Omega \subset$ $\left[c_{1}, d_{1}\right]$ such that mes $\Omega>0$ and

$$
\int_{h(t)}^{\Delta(t)} K(t, s) y(s) d s>0 \text { for a.e. } t \in \Omega .
$$

Then

$$
\int_{h(t)}^{\Delta(t)} K(t, s) y(s) d s>0 \text { for a.e. } t>d_{1}
$$

Proof of Lemma 4.1. Rewrite inequality (4.1) in the form

$$
-\int_{h(t)}^{a} K(t, s) y(s) d s<\int_{a}^{\Delta(t)} K(t, s) y(s) d s, \text { for a.e. } t \in \Omega
$$

Giving an increment $\tau>0$, we obtain

$$
-\int_{h(t+\tau)}^{a} K(t+\tau, s) y(s) d s=-\int_{h(t+\tau)}^{a} A(t, \tau, s) K(t, s) y(s) d s \leq-\int_{h(t)}^{a} A(t, \tau, s) K(t, s) y(s) d s .
$$

Using nondecreasing $A(t, \tau, \cdot)$ we can continue

$$
\begin{aligned}
& -\int_{h(t)}^{a} A(t, \tau, s) K(t, s) y(s) d s<\int_{a}^{\Delta(t)} A(t, \tau, s) K(t, s) y(s) d s \leq \\
& \leq \int_{a}^{\Delta(t+\tau)} A(t, \tau, s) K(t, s) y(s) d s=\int_{a}^{\Delta(t+\tau)} K(t+\tau, s) y(s) d s .
\end{aligned}
$$

Thus

$$
-\int_{h(t+\tau)}^{a} K(t+\tau, s) y(s) d s<\int_{a}^{\Delta(t+\tau)} K(t+\tau, s) y(s) d s .
$$

This completes the proof of inequality (4.2) and Lemma 4.1.

Proof of Theorem 3.1. Consider a solution $x(t)$ of equation (3.1). Let us suppose that $\alpha_{1}$ is its first zero in $(0, \infty)$. This zero cannot be multiple. Actually, if we assume, for example, that $x(t)<0$ for $t \in\left[0, \alpha_{1}\right)$, then there exists a point $t_{1} \in\left(0, \alpha_{1}\right)$ such that $x^{\prime}\left(t_{1}\right)>0$. From equation (3.1), we obtain

$$
\left.x^{\prime}\left(\alpha_{1}\right)=x^{\prime}\left(t_{1}\right)+\int_{t_{1}}^{\alpha_{1}} x^{\prime \prime}(s)\right) d s=x^{\prime}\left(t_{1}\right)-\int_{t_{1}}^{\alpha_{1}} \int_{h(s)}^{\Delta(s)} K(s, \xi) x(\xi) d \xi d s>0 .
$$


Denote $\beta_{1}$ the first after $\alpha_{1}$ zero of the derivative $x^{\prime}(t)$. If there is no such $\beta_{1}$, then the theorem has been proven. Let, for example, $x^{\prime}\left(\alpha_{1}\right)>0$. There exists the set $\Omega_{1} \subset\left(\alpha_{1}, \beta_{1}\right)$ such that

$$
\int_{h(t)}^{\Delta(t)} K(t, s) x(s) d s>0 \text { for a.e. } t \in \Omega_{1}
$$

and $m e s \Omega_{1}>0$. If not, we have

$$
\left.x^{\prime}(t)=x^{\prime}\left(\alpha_{1}\right)+\int_{\alpha_{1}}^{t} x^{\prime \prime}(s)\right) d s=x^{\prime}\left(\alpha_{1}\right)-\int_{\alpha_{1}}^{t} \int_{h(s)}^{\Delta(s)} K(s, \xi) x(\xi) d \xi d s>0 \text { for } t \geq \alpha_{1}
$$

and there is no the point $\beta_{1}$, where $x^{\prime}\left(\beta_{1}\right)=0$. Now by Lemma 4.1 we obtain

$$
\int_{h(t)}^{\Delta(t)} K(t, s) x(s) d s>0 \text { for a.e. } t \in\left[\beta_{1}, \alpha_{2}\right],
$$

where $\alpha_{2}$ the following after $\alpha_{1}$ zero of solution $x(t)$. It is clear that there exists a point $\gamma_{1} \in\left(\beta_{1}, \alpha_{2}\right)$ such that $x^{\prime}\left(\gamma_{1}\right)<0$. We obtain

$$
x^{\prime}\left(\alpha_{2}\right)=x^{\prime}\left(\gamma_{1}\right)+\int_{\gamma_{1}}^{\alpha_{2}} x^{\prime \prime}(s) d s=x^{\prime}\left(\alpha_{1}\right)-\int_{\gamma_{1}}^{\alpha_{2}} \int_{h(s)}^{\Delta(s)} K(s, \xi) x(\xi) d \xi d s<0 .
$$

Let us prove that $\rho\left(K_{\alpha_{1} \alpha_{2}}\right) \geq 1$. The solution $x(t)$ satisfies for $t \in\left[\alpha_{1}, \alpha_{2}\right]$ the following equation

$$
x(t)=-\int_{\alpha_{1}}^{\alpha_{2}} G_{\alpha_{1} \alpha_{2}}(t, s) \int_{h(s)}^{\Delta(s)} K(s, \xi) x(\xi) d \xi d s,
$$

where $G_{\alpha_{1} \alpha_{2}}(t, s)$ is defined by (2.2). It follows from (4.8) that $\Delta(t)>\alpha_{1}$ for $t \in\left[\beta_{1}, \alpha_{2}\right]$. Equation (4.10) can be rewritten in the form

$$
x(t)=-\int_{\alpha_{1}}^{\alpha_{2}} G_{\alpha_{1} \alpha_{2}}(t, s)\left\{\int_{h(s)}^{\alpha_{1}} K(s, \xi) x(\xi) d \xi+\int_{\alpha_{1}}^{\Delta(s)} K(s, \xi) x(\xi) d \xi\right\} d s .
$$

Using the fact that $\alpha_{1}$ cannot be a multiple zero, we have $x(t)>0$ for $t \in\left(\alpha_{1}, \alpha_{2}\right), x(t)<0$ for $t \in\left(0, \alpha_{1}\right)$. Setting $v(t)=x(t)$ in Lemma 2.2, we obtain that $\rho\left(K_{\alpha_{1} \alpha_{2}}\right) \geq 1$. Let $\beta_{2}$ and $\alpha_{3}$ are following after $\alpha_{2}$ zeros of the derivative $x^{\prime}(t)$ and the solution $x(t)$ respectively. If the solution does not have them, the proof is completed.

Let us demonstrate that $\Delta(t)>\alpha_{2}$ for $t \in\left[\beta_{2}, \alpha_{3}\right]$. Actually, there exists the set $\Omega_{2} \subset\left(\alpha_{2}, \beta_{2}\right)$ such that

$$
\int_{h(t)}^{\Delta(t)} K(t, s) x(s) d s<0 \text { for a.e. } t \in \Omega_{2}
$$

and $m e s \Omega_{2}>0$. If not, we have

$$
x^{\prime}(t)=x^{\prime}\left(\alpha_{2}\right)+\int_{\alpha_{2}}^{t} x^{\prime \prime}(s) d s=x^{\prime}\left(\alpha_{1}\right)-\int_{\alpha_{2}}^{t} \int_{h(s)}^{\Delta(s)} K(s, \xi) x(\xi) d \xi d s<0 \text { for } t \geq \alpha_{2},
$$

and there is no the point $\beta_{2}$ such that $x^{\prime}\left(\beta_{2}\right)=0$.

Now the condition $\rho\left(K_{h(t) \Delta(t)}\right)<1$ for $t \in(0, \infty)$ and the proven above fact that $\rho\left(K_{\alpha_{1} \alpha_{2}}\right) \geq 1$ imply, according to Lemma 2.4, that $h(t)>\alpha_{1}$ for a.e. $t \in\left[\beta_{2}, \alpha_{3}\right]$. Now Lemma 4.1 implies that

$$
\int_{h(t)}^{\Delta(t)} K(t, s) x(s) d s<0 \text { for a.e. } t \in\left[\beta_{2}, \alpha_{3}\right] \text {. }
$$


According to the La Grange theorem, there exists a point $\gamma_{2} \in\left(\beta_{2}, \alpha_{3}\right)$ such that $x^{\prime}\left(\gamma_{2}\right)<0$ and

$$
\left.x^{\prime}\left(\alpha_{3}\right)=x^{\prime}\left(\gamma_{2}\right)+\int_{\gamma_{2}}^{\alpha_{3}} x^{\prime \prime}(s)\right) d s=x^{\prime}\left(\alpha_{1}\right)-\int_{\gamma_{2}}^{\alpha_{3}} \int_{h(s)}^{\Delta(s)} K(s, \xi) x(\xi) d \xi d s<0 .
$$

We have proven that the zero $\alpha_{3}$ cannot be multiple.

Now we have to prove that $\rho\left(K_{\alpha_{2} \alpha_{3}}\right) \geq 1$. The solution $x(t)$ satisfies for $t \in\left[\alpha_{2}, \alpha_{3}\right]$ the following equation

$$
x(t)=-\int_{\alpha_{2}}^{\alpha_{3}} G_{\alpha_{2} \alpha_{3}}(t, s) \int_{h(s)}^{\Delta(s)} K(s, \xi) x(\xi) d \xi d s .
$$

where $G_{\alpha_{2} \alpha_{3}}(t, s)$ is defined by (2.2). It follows from (4.11) that $\Delta(t)>\alpha_{2}$ for $t \in\left[\beta_{2}, \alpha_{3}\right]$. Equation (4.15) can be rewritten in the form

$$
x(t)=-\int_{\alpha_{2}}^{\alpha_{3}} G_{\alpha_{2} \alpha_{3}}(t, s)\left\{\int_{h(s)}^{\alpha_{2}} K(s, \xi) x(\xi) d \xi+\int_{\alpha_{2}}^{\Delta(s)} K(s, \xi) x(\xi) d \xi\right\} d s .
$$

Let us prove that

$$
\int_{h(t)}^{\alpha_{2}} K(t, s) x(s) d s \geq 0 \text { for a.e. } t \in\left[\alpha_{2}, \alpha_{3}\right] .
$$

We proved above that $h(t)>\alpha_{1}$ for a.e. $t \geq \beta_{2}$. But for $t \in\left[\alpha_{2}, \beta_{2}\right]$ the inequality $h(t)<\alpha_{1}$ is possible. Let us set $s=\theta+\tau$. We proved above that there exists the set $\Omega_{1} \subset\left(\alpha_{1}, \beta_{1}\right)$ such that

$$
\int_{h(\theta)}^{\Delta(\theta)} K(\theta, \xi) x(\xi) d \xi>0 \text { for } \theta \in \Omega_{1},
$$

and $m e s \Omega_{1}>0$. Using nondecreasing of $A(\theta, \tau, \cdot)$, we can write

$$
0<\int_{h(\theta)}^{\Delta(\theta)} A(\theta, \tau, \xi) K(\theta, \xi) x(\xi) d \xi \leq \int_{h(s)}^{\Delta(s)} K(s, \xi) x(\xi) d \xi \leq \int_{h(s)}^{\alpha_{2}} K(s, \xi) x(\xi) d \xi .
$$

Thus we have proven (4.17).

Now we can set $v(t)=-x(t)$ in the condition of Lemma 2.2 and obtain, according to this lemma, that $\rho\left(K_{\alpha_{2} \alpha_{3}}\right) \geq 1$. Let $\left\{\alpha_{n}\right\}, n=1,2,3, \ldots$ are series of zeros of the solution $x(t)$. Repeating our reasoning, we can prove that there are no multiple zeros and $\rho\left(K_{\alpha_{n} \alpha_{n+1}}\right) \geq 1$. From this we have also the inequality

$$
\alpha_{n+1}-\alpha_{n}>\frac{4}{\int_{\alpha_{n}}^{\alpha_{n+1}} \int_{\alpha_{n}}^{s} K(s, \xi) d \xi d s} \geq \frac{4}{\int_{0}^{\alpha_{n+1}} \int_{0}^{s} K(s, \xi) d \xi d s},
$$

demonstrating a possibility of existence of only finite number of zeros on every finite interval. It explains that all zeros of the solution $x(t)$ are in this series. This completes the proof.

\section{REFERENCES}

[1] R.P. Agarwal, L. Berezansky, E. Braverman, A. Domoshnitsky, Nonoscillation Theory of Functional Differential Equations with Applications, Springer, New York, 2012.

[2] N.V. Azbelev, About zeros of solutions of linear differential equations of the second order with delayed argumen, Differentsialnye Uravnenia, 7 (1971), 1147-1157.

[3] N.V. Azbelev, V.P. Maksimov, L.F. Rakhmatullina, Introduction to theory of functional-differential equations, Nauka, Moscow, 1991.

[4] A. Domoshnitsky, Extension of Sturm's theorem to apply to an equation with time-lag, Differential Equations, 19 (1983), 1099-1105. Translation from Differentsial'nye Uravnenia, 19 (1983), 1475-1482.

[5] A. Domoshnitsky, Unboundedness of solutions and instability of differential equations of the second order with delayed argument, Differential \& Integral Equations, 14 (2001), 559-576 
[6] A. Domoshnitsky, Wronskian of fundamental system of delay differential equations, Funct. Differ. Equ. 9 (2002), $353-$ 376.

[7] A.Domoshnitsky. Sturm's theorem for equation with delayed argument, Georgian Math. J. 1 (1994), 267-276.

[8] A. Domoshnitsky, One approach to analysis of asymptotic and oscillation properties of delay and integral PDE, Dyn. Contin. Discrete Impuls. Syst. Ser. A Math. Anal. 12 (2005), 263-274.

[9] Y. Domshlak, Comparison theorems of Sturm type for first and second differential equations with sign variable deviations of the argument, Ukraine Mat.Zh. 34 (1982), 158-163.

[10] S.V. Eliason, Distance between zeros of certain differential equations having delayed arguments, Ann. Math. Pura Appl. 106 (1975), 273-291.

[11] L.N. Erbe, Q. Kong, B.G. Zhang, Oscillation theory for functional differential equations, Dekker, New York/Basel, 1995.

[12] I. Gyori, G.Ladas, Oscillation theory of delay differential equations, Clarendon, Oxford, 1991.

[13] P. Hartman, Ordinary differential equations, Moscow, Nauka, 1970.

[14] Yu.V. Komlenko, Sufficient conditions of regularity of periodic problem for Hill's equation with deviating argument, In book: Mathematical Physics, Kiev, pp. 5-12, 1977.

[15] R.G. Koplatadze, On oscillatory properties of solutions of functional differential equations, Mem. Differential Equations Math. Phys. 3 (1994), 2-179.

[16] R. Koplatadze, G. Kvinikadze, I.P. Stavroulakis, Oscillation of second order lonear delay differential equations, Functional Differential Equations, 7 (2000), 121-145.

[17] M. A. Kranoselski, Approximate Solution of Operator Equations, D. Wolters-Noordhoff Pub, Groningen, 1972.

[18] S.M. Labovskii, About properties of fundamental system of solutions of a second order differential equations with delayed argument, Trudy TIHM, 6 (1971), 49-52 (in Russian).

[19] S.M. Labovskii, Condition of nonvanishing of Wronskian of fundamental system of linear equation with delayed argument, Differentsial'nye Uravnenia, 10 (1974), 426-430.

[20] G.S. Ladde, V. Lakshmikantham, B.G. Zhang, Oscillation theory of differential equations with deviating argument, Dekker, New York/Basel, 1987.

[21] A.D. Myshkis, Linear differential equations with delayed argument, Moscow, Nauka, 1972.

[22] S.B. Norkin, Differential equations of the second order with retarded argument, Transl. Math. Monographs, Volume 33, Amer. Math. Soc., Moscow, 1972.

[23] D.V. Paatashvili, About unbounded solutions of linear differential equations of second order with delay argument, Differentsial'nye Uravnenia, 25 (1989), 774-780.

[24] X. Zhou, The estimate for number of zeros of solutions of second order functional differential equations, Appl. Math. Comput. 215 (2009), 1266-1273.

[25] N.E. Zhukovskii, Complete Collected Works, Common Mechanics (Moscow, Leningrad, 1937) (in Russian). 